\title{
A framework for multiple moving objects detection in aerial videos
}

\begin{abstract}
Aerial videos captured using dynamic cameras commonly require background remodeling at every frame. In addition, camera motion and the movement of multiple objects present an unstable imaging environment with varying motion patterns. This makes detecting multiple moving objects a difficult task. In this chapter, a two-step framework, termed the motion differences of matched region-based features (MDMRBF), is presented. Firstly, each frame goes through super-pixel segmentation to produce regions where each frame is then represented as a region adjacency graph structure of visual appearance and geometric properties. This representation is important for correspondence discovery between consecutive frames based on multigraph matching. Ultimately, each region is labeled as either a background or foreground (object) using a proposed graph-coloring algorithm. Two datasets, namely (1) the DARPA-VIVID dataset and (2) self-captured videos using an unmanned aerial vehiclemounted camera, have been used to validate the feasibility of MDMRBF. Comparison is also done with three existing detection algorithms where experiments show promising results with precision at $94 \%$, and recall at $89 \%$.
\end{abstract}

Keyword: Motion similarity graph; Moving object detection; Multigraph matching; Unmanned aerial vehicle (UAV) 